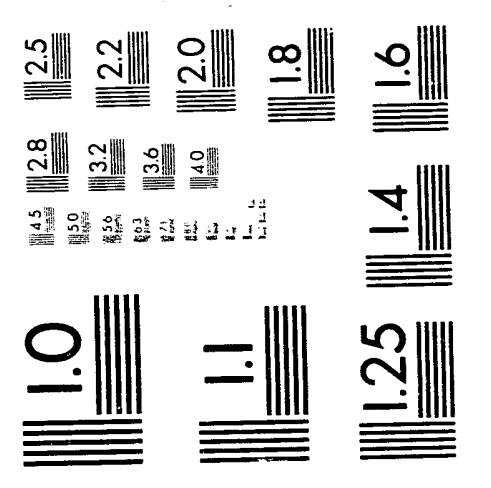



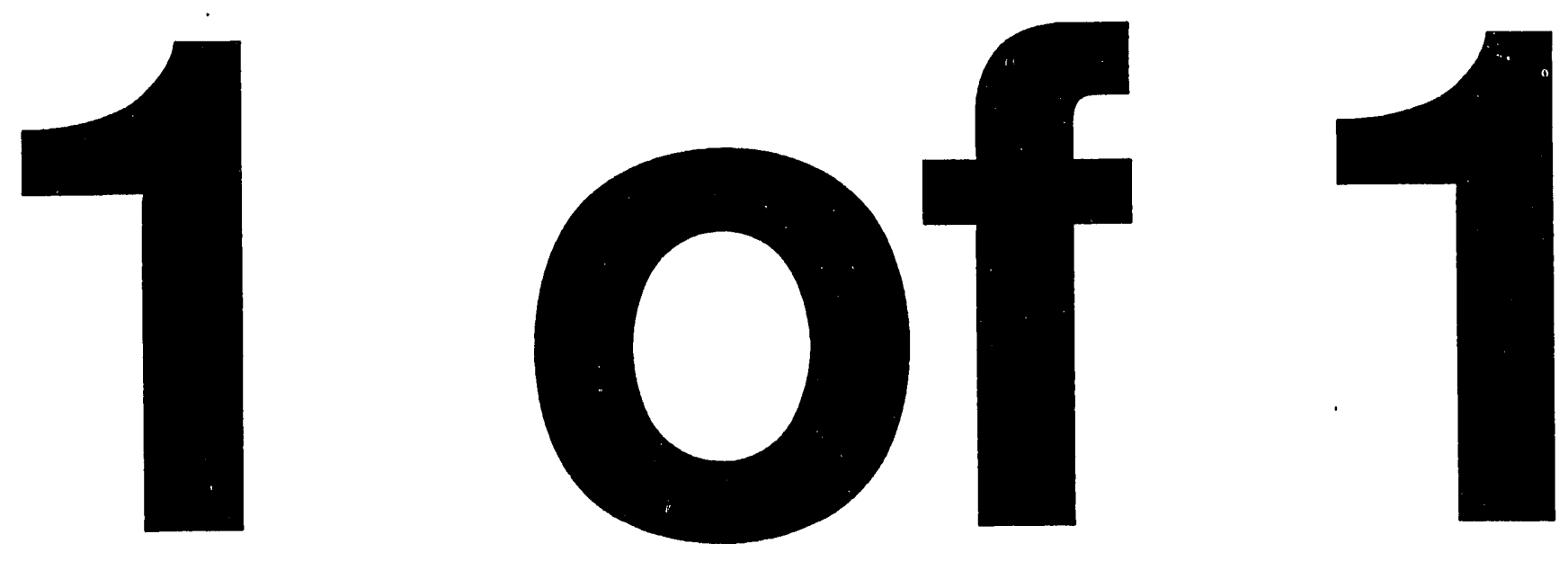


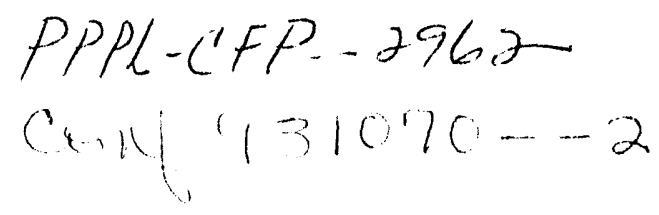

\title{
Neutral Gas Transport Modeling with DEGAS $2^{(1}$
}

\author{
Daren Stotler and Charles Karney \\ Plasma Physics Laboratory, Princeton University \\ Princeton, NJ, USA
}

\begin{abstract}
We are currently rewriting the neutral gas transport code, DEGAS $[1,2]$, with a view to not only making it faster, but also easing the process of including new physics. The goal is to make adding new species and reactions relatively simple so that the code can be rapidly adapted to new divertor physics regimes. DEGAS 2 will also be optimized for coupling to fluid plasma codes, incorporating many of the techniques utilized in B2-EIRENE[3-5]. Finally, it is our intention that DEGAS 2, like DEGAS, be well-documented and easy to use. We will present model calculations including ionization and charge exchange which will illustrate the way reactions are included into DEGAS 2 and will demonstrate operation of the code on a distributed network of workstations.
\end{abstract}

\section{Introduction}

The DEGAS Monte Carlo neutral transport code[1,2] is in wide use around the world. Its flexibility, ease-of-use, and documentation account in large part for its popularity. Now that the coupling of Monte Carlo neutral and fluid plasma transport codes has become practical[4,5], however, it is time to reconsider the algorithms on which DEGAS is based. Namely, DEGAS is far too slow, particularly in high recycling regimes, to allow it to be coupled effectively to a fluid code where hundreds or even thousands of complete Monte Carlo profile calculations must be carried out. This problem will be addressed by replacing the pseudo-collision algorithm $|1,2|$ in DEGAS with a track-length estimator, allowing DEGAS 2 to deal effectively with the wide density ranges (vacuum to $10^{21} \mathrm{~m}^{-3}$ ) anticipated in high recycling divertors. Associated with this change is the usage of a different approach to handling the geometry; the new representation will allow DEGAS 2 to easily handle complex (realistic) device configurations.

Furthermore, adding new physics (e.g., new neutral species and reactions) to DEGAS is somewhat cumbersome. This is again a concern in the high recycling regime where we anticipate that new types of physics (elastic collisions, molecular hydrogenic species other than $\mathrm{H}_{2}$ ) may be important. DEGAS 2 will utilize an object-oriented approach to organize the atomic and surface physics data, facilitating changes. Another motivation for doing this is to allow the code to be run with a minimal set of reactions, e.g., for exploratory calculations or preliminary iterations with a fluid code.

A number of techniques, many developed for the B2-EIRENE code[3-5], will be employed to make the coupling of neutral and fluid plasma codes as efficient as possible. DEGAS 2 will also be able to make effective use of massively parallel computers as they become available.

As we are rewriting the code, we intend to retain the overall ease-of-use and portability of DEGAS. We are also documenting the code as it is being written via the WE B package[10], interleaving code and $(T E X)$ documentation. Furthermore, the C-like preprocessing capabilities provided by WEB for FORTRAN have enabled us to implement in a fairly natural way the object-oriented programming techniques discussed in this paper

\footnotetext{
(15ubmitted to the 4th International Workshop on Plasma Edge Theory in Fusion Devices, 4 - 6 October 1993, 
This paper is organized as follows. The techniques used for geometry and tracking are described in Sec. 2. Particles and reactions are discussed in Sec. 3; this section includes as well an example run of DEGAS 2 in its present form. Section 4 details some of the techniques for coupling to plasma calculations to be installed in DEGAS 2. The computational features of DEGAS 2 are described in Sec. 5.

\section{Geometry and Tracking}

The problem space in DEGAS 2 is divided into "zones" in which properties are constant (e.g., a plasma element, vacuum region, or divertor plate). The connectivity of the zones is arbitrary, allowing complex geometries, such as general double null plasmas or devices with complicated baffling structures, to be simulated. Each zone is subdivided by the code into "pseudo-convex" cells defined by quadratic surfaces $f_{i j}(\vec{x})=0$, with $i$ being the cell number and $j$ numbering the surfaces for that cell,

$$
C_{i}=\bigcap_{j}\left\{\vec{x}: f_{i j}(\vec{x}) \geq 0\right\}
$$

DEGAS 2 maintains a list of cells on the positive and negative sides of each surface $f_{i j}$.

The tracking of a flight within a cell is done by computing the intersection of its path with each surface of the cell. The proper path is the one with the earliest (in time of flight) intersection; the identity of the succeeding cell is determined from the list of neighboring cells.

For neutral species, the path of the flight is simply given by

$$
\vec{x}_{\boldsymbol{i}+1}=\vec{x}_{\boldsymbol{i}}+\vec{v} t
$$

DEGAS 2 will also be capable of tracking charged particles along field lines. For this purpose, a toroidally axisymmetric field

$$
\vec{B}=\nabla \psi \times \nabla \zeta+F(\psi) \nabla \zeta,
$$

where $\psi$ is the poloidal flux and $\zeta$ is the toroidal angle, is assumed. A linear fit to $\psi$,

$$
\psi=\psi_{0}+a R+b z
$$

where $R$ and $z$ are the radial and vertical coordinates, can be used to track the flight within each cell. This scheme will be generalized to handle a general equation of motion through a simple ordinary differential equation solver.

\section{Particles and Reactions}

We have used object-oriented programming techniques in DEGAS 2 to enhance the flexibility of the atomic and surface physics in the code, making it easy to add or remove species, reactions etc. We have also placed many of the physics details into external files, providing one with a succinct description of the physics used in a particular calculation. We discuss here only the treatment of the atomic physics; the surface physics will be implemented in an analogous fashion.

Each atomic or molecular "species" is composed of "elements", a list which corresponds to the elements of the periodic table but includes as well electrons, photons, and whatever other fundamental constituents are necessary for the calculation. Tables I and II give some examples of elements and species, respectively.

A particle (or flight) in DEGAS 2 has several attributes. The first is its species. Others are associated with tracking: accumulated time, velocity, and position. The particle also has a statistical weight (in practice, the flux associated with the particle) which is used in scoring. 


\begin{tabular}{|c|c|c|l|}
\hline Symbol & $\mathrm{Z}$ & Mass & Name \\
\hline \hline $\mathbf{e}$ & -1 & $1 / 1840$ & electron \\
\hline $\mathrm{H}$ & 1 & 1 & hydrogen \\
\hline $\mathrm{D}$ & 1 & 2 & deuterium \\
\hline $\mathrm{He}$ & 2 & 4 & helium \\
\hline
\end{tabular}

Table I: Abbreviated list of "elements" in DEGAS 2

\begin{tabular}{|c|c|}
\hline Symbol & Composition \\
\hline $\mathbf{e}$ & $\mathbf{e}$ \\
\hline $\mathrm{H}$ & $\mathrm{H}$ \\
\hline $\mathrm{H}_{2}$ & $\mathrm{H}+\mathrm{H}$ \\
\hline $\mathrm{H}^{+}$ & $\mathrm{H}-\mathrm{e}$ \\
\hline
\end{tabular}

Table II: Examples of particle compositions in terms of "elements".

Background species form a separate class. Each instance of this class also has as an attribute its species; its density, temperature, and flow velocity fields (as functions of zone number) are additional attributes.

The reactions considered by DEGAS 2 form another class. Each reaction has as attributes the number of reagents, the number of products, and the species of each of these. On input, this information is specified in an obvious manner. For example, the reaction $\mathrm{H}+\mathrm{He}^{+} \rightarrow \mathrm{H}^{+}+\mathrm{He}$ might be identified in ihe input file as

$$
\mathrm{H}+\mathrm{He}+\rightarrow \mathrm{H}++\mathrm{He} \text {. }
$$

The input information will further specify a subroutine responsible for reading and evaluating the reaction rate data. A separate subroutine, specific to the type of reaction (charge exchange, ionization, etc.) will be used to set the velocities and weights of the reaction products. This approach is similar to, and will hopefully be compatible with, ALADDIN $[6]$ data representation techniques. As in DEGAS, reactions need to be provided only for one isotope of a species; rates for isotopic variants can be obtained by rescaling temperatures and energies to match the velocity of the reference isotope.

Each particle will have associated with it a list of valid reactions in which it can participate. A valid reaction will have the particle species as one of its reagents; the other reagents in that reaction must be present in the list of background particles. During the tracking of a given particle, the code would loop through this list in order to determine which of the possible reactions is to occur next.

\section{Coupling to Fluid Codes}

The techniques employed in DEGAS 2 to optimize the coupling to fluid plasma codes have their basis in the work done in coupling B2[3] with EIRENE[4,5] and DEGAS[7]. The first of these addresses the issue of statistical errors in Monte Carlo results. In a fluid code, the plasma variables change slightly from time-step to time-step. Ideally, one would like to have the source rates due to neutrals respond precisely to each of these variations. In a Monte Carlo calculation, however, the changes in the source rates will be convoluted with some level of random noise. When changes in the plasma parameters between runs are small, this noise can dominate the system and prevent the codes from converging. It is possible to reduce everywhere the noise level by adding more neutral flights, but the resulting run time is prohibitively long. 17]

The technique of correlated sampling $[8]$ eliminates this problem. The particle tracks are computed using some reference plasma with density and temperature profiles $n_{0}(\vec{x}), T_{0}(\vec{x})$. In subsequent Monte Carlo runs for plasmas $n_{0}(\vec{x})+\delta n(\vec{x}), T_{0}(\vec{x})+\delta T(\vec{x})$, DEGAS 2 computes scores based on these tracks hut writh monlifiod wointe 


$$
w=\frac{\langle\sigma v\rangle_{n_{0}+\delta n_{1}, T_{0}+\delta T}}{\langle\sigma v\rangle_{\left.n_{0}, T_{0}\right)}}
$$

The Monte Carlo solution still has an error proportional to $1 / \sqrt{N}$ where $N$ is the number of flights, but this error is correlated with that in the initial calculation so that the fluid code does not see a fluctuating source.

The handling of high-recycling situations is facilitated by an implicit coupling procedure $[4,5]$ in which the fluid code updates at each of its time steps the source rates due to neutrals, using the reaction rates from the neutral transport code to account for changes in the plasma parameters. Once the fluid code iterations have progressed to the point where the integrated particle and energy fluxes to the target have changed by some predetermined amount (usually a few percent), the Monte Carlo code is called again. The technique of averaging source rates[7] from consecutive neutral calculations should also aid convergence in high recycling simulations.

\section{Numerical and Computational Considerations}

The most promising computational technique for improving the speed of the Monte Carlo calculation is the use of parallel processing; the original version of DEGAS $[1,2]$ incorporated this as well. Parallel processing is practical in a Monte Carlo calculation since the amount of communication required per computation is small. We feel that it is essential to maximize the parallel processing capability in DEGAS 2 since most of the future advancements in computer speed will result from increases in the number of processing units.

Parallel computing will be implemented in DEGAS 2 via the Parallel Virtual Machine (PVM) software[9]. The basic strategy is embodied in the following steps:

- Master process is started.

- Master process starts up slave processes.

- Master distributes requests for flight computations to slaves.

- Each slave, when it is done, sends the results back to the master and receives a new request.

One of the virtues of PVM is that it works across different machine arch.tectures as well as on parallel processing devices. Furthermore, it is possible to set up PVM to perform automatic load balancing so that faster or less used machines do more of the work.

The use of multiple processors on different architectures such as is done with PVM makes reproducibility, desirable for debugging difficult. To maximize reproducibility, we first use a pseudo-random number generator which gives the same sequence on different machines. Secondly, each flight $k$ is assigned its own pseudo-random number seed $S_{k}$ so that minor variations in one flight will not impact the random number sequence of a subsequent flight.

\section{Conclusions}

We have described the present status of the DEGAS 2 code work. The tracking and geometry routines are complete and working. The framework for handling particles and reactions is finished; examples using a few of the most basic reactions have been given. Implementation of scoring and surface physics will follow in the near future, as will other detailed features. We are hopeful that DEGAS 2 will provide as much insight into neutral gas physics (and other topics as well) over the next decade as DEGAS has in the past. 


\section{Acknowledgments}

The authors wish to thank $\mathrm{D}$. Reiter and $\mathrm{R}$. Schneider for providing many useful discussions. Of course, DEGAS 2 would not be possible were it not for the pioneering efforts of D. Heifetz and the other authors of DEGAS. This work was supported by U.S. DOE Contract No. DE-AC02-76-CHO-3073.

\section{References}

[1] Heifetz, D., Post, D., Petravic, M., Weisheit, J., and Bateman, G., J. Comp. Phys., 46 (1982) 309.

[2] Heifetz, D. B. in: Physics of Plasma-Wall Interactions in Controlled Fusion, Series B, Physics vol. 131, NATO ASI Series, eds. D. E. Post and R. Behrisch (Plenum, Quebec, 1984).

[3] Braams, B. J., Computational Studies in Tokamak Equilibrium and Transport, Thesis (University of Utrecht, 1986).

[4] Reiter, D., Kever, H., Wolf, G. H., Baelmans, M. Behrisch, R. and Schneider, R., Plasma Phys. and Controlled Fusion 33 (1991) 1579.

[5] Schneider, R., Reiter, D., et al., J. Nucl. Mat. 196-198 (1992) 810.

|6| Hulse, R. A., in AIP Conf. Proceedings 206: Atomic Processess in Plasmas, (American Institute of Physics, New York, 1990).

17| Stototer, D. P., Cos'ter, D. P. et al., J. Nucl. Mat. 196-198 (1992) 894.

[8] Spanier, J. and Gelbard, E. M., Monte Carlo Principles and Neutral Transport Problems (Addison-Wesley Publishing Company, Reading, Massachusetts, 1969).

[9] Sunderam, V., Concurrency: Practice and Experience, 2 (1990) 315.

[10] Krommes, J., Private Communication, Princeton University, Plasma Physics Laboratory.

\section{DISCLAIMER}

This report was prepared as an account of work sponsored by an agency of the United States Government. Neither the United States Government nor any agency thereof, nor any of their employees, makes any warranty, express or implied, or assumes any legal liability or responsibility for the accuracy, completeness, or usefulness of any information, apparatus, product, or process disclosed, or represents that its use would not infringe privately owned rights. Reference herein to any specific commercial product, process, or service by trade name, trademark, manufacturer, or otherwise doses not necessarily constitute or imply its endorsement, recommendation, or favoring by the United States Government or any agency thereof. The views and opinions of authors expressed herein do not necessarily state or reflect those of the United States Government or any agency thereof. 
